\title{
Bounding Network-Induced Delays for Time-Critical Services in Avionic Systems Using Measurements and Network Calculus
}

\author{
Huan Yang \\ Department of Computer Science and \\ Engineering \\ Lehigh University \\ Bethlehem, Pennsylvania \\ huy213@lehigh.edu
}

\author{
Liang Cheng \\ Department of Computer Science and \\ Engineering \\ Lehigh University \\ Bethlehem, Pennsylvania \\ cheng@cse.lehigh.edu
}

\author{
Xiaoguang Ma \\ Department of Electrical Engineering \\ University of Wisconsin-Platteville \\ Platteville, Wisconsin \\ max@uwplatt.edu
}

\begin{abstract}
In this work, we propose to derive realistic, accurate bounds on network-induced delays for time-critical tasks running on Avionics Full-Duplex Switched (AFDX) Ethernet. Our preliminary evaluation results show that through measurement-based modeling and refining network-calculus-based analysis with measurements tight delay bounds can be obtained for AFDX networks with realistic traffic patterns and network workloads.
\end{abstract}

ACM Reference Format:

Huan Yang, Liang Cheng, and Xiaoguang Ma. 2019. Bounding NetworkInduced Delays for Time-Critical Services in Avionic Systems Using Measurements and Network Calculus. In ICCPS '19: ACM/IEEE International Conference on Cyber-Physical Systems, April 16-18, 2019, Montreal, QC, Canada. ACM, New York, NY, USA, 2 pages. https://doi.org/10.1145/3302509.3313330

\section{INTRODUCTION}

As the de-facto standard for the transmission of network traffic flows of critical avionics applications, Avionics Full-Duplex Switched (AFDX) Ethernet interconnects end systems (e.g., sensor and actuator nodes) with Ethernet switch(es) based on First-in Firstout (FIFO) scheduling [2]. For such a networked cyber-physical system (NCPS), it is of vital importance to guarantee that stringent real-time requirements of critical avionics applications are met. Therefore, an analytical tool facilitating the evaluation of worstcase delay performance (i.e., the worst-case end-to-end communication delays experienced by all time-critical flows) of practical AFDX networks is urgently needed.

As a theoretical tool for worst-case delay analysis, network calculus has been applied to FIFO networks to derive formally provable delay bounds [1]. However, specification-based network-calculus models (e.g., constructed based on sampling rates and data formats of source end systems) are typically adopted during analysis, and little has been done to validate existing analytical methods in real-world AFDX networks. In fact, it is shown in [3] that delay bounds obtained from network-calculus-based analysis can be further tightened through taking network measurements. To evaluate whether network-calculus-based analysis can provide accurate delay bounds for practical AFDX networks, we combine network measurements with network-calculus-based analysis in

Permission to make digital or hard copies of part or all of this work for personal or classroom use is granted without fee provided that copies are not made or distributed for profit or commercial advantage and that copies bear this notice and the full citation on the first page. Copyrights for third-party components of this work must be honored

For all other uses, contact the owner/author(s).

ICCPS '19, April 16-18, 2019, Montreal, QC, Canada

(c) 2019 Copyright held by the owner/author(s).

ACM ISBN 978-1-4503-6285-6/19/04 . \$ \$15.00

https://doi.org/10.1145/3302509.3313330

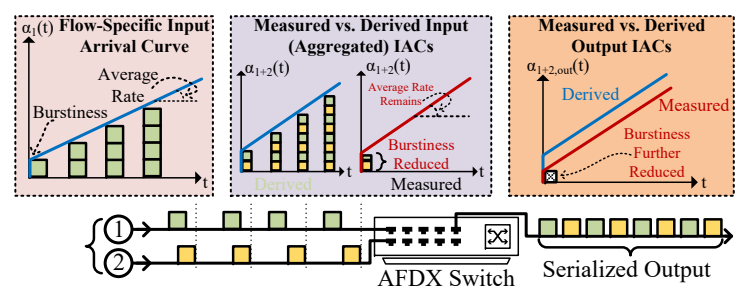

Figure 1: Measurement-based vs. derived models.

this work and examine the quality of delay bounds obtained under application-specific traffic patterns of AFDX systems. By modeling traffic sources and networking devices (e.g., Ethernet switches) through measurements, we establish accurate, realistic traffic and device models for network-calculus-based analysis. Using our approach, different network-calculus-based analytical methods can be leveraged, allowing AFDX architects to construct a proper delay analysis framework to suit application-specific needs (e.g., balancing between computational complexity and tightness of bounds).

In this work, we use the optimization-based analytical approach proposed in [1] and the measurement method outlined in [3]. Our preliminary results show that analytical bounds can be further improved for practical AFDX networks by plugging in measurementbased models and replacing intermediate arrival curves (IACs) with those constructed from measurements.

\section{APPROACH}

Given the traffic pattern of an AFDX network, our approach first extracts traffic characteristics of source end systems and establishes their respective traffic models. Then, we inject packets based on application-specific requirements (e.g., packet sizes) to model AFDX Ethernet switches. Finally, worst-case delay analysis is performed by properly supplying existing analytical method(s) with measurement-based models.

\subsection{Modeling Source End Systems}

To perform network-calculus-based analysis, arrival curves of source end systems and service curves of FIFO Ethernet switches need to be established. To construct the arrival curve of an end system (e.g., a leaky-bucket curve $\alpha(t)=\sigma+\rho \cdot t$, with $\sigma$ being the burstiness component and $\rho$ representing the average rate), we use netFPGA [4] to capture and timestamp its network packets. Note that if actual measurements cannot be directly taken (e.g., in early stage of AFDX design where source end systems have not been procured), we can emulate the source end system of interest based on its specifications (e.g., sampling rates of sensors and minimum inter-arrival time imposed by traffic shaping techniques). Then, burstiness and average 
rate can be found by applying the definition of arrival curve:

$$
\forall t \geq s \geq 0: \alpha(t-s) \geq F(t)-F(s),
$$

where $F(t)$ is the cumulative traffic volume seen from the source end system up to time instant $t$.

\subsection{Identifying Measurement-Based Intermediate Arrival Curves}

In existing analytical methods, intermediate arrival curves (IACs) for traffic flows seen at Ethernet switches must be properly characterized through measurements (e.g., using netFPGA and Ethernet tap). As shown in Figure 1, by taking into account serialization effects at any output interface of the switch, burstiness component of the output flow can be reduced. In addition, statistical multiplexing among source end systems may also result in reduced burstiness (at the input end). Therefore, measurement-based models should be properly integrated into existing analytical methods.

\subsection{Modeling Ethernet Switches}

To obtain service curve of an Ethernet switch (e.g., a rate-latency curve $\beta(t)=\max \{0, R \cdot(t-T)\}$, with $R$ being the processing rate and $T$ representing the latency component), rate component can be determined from switch specifications (e.g., if the flow of interest passes through a switch with 100-Mbps interfaces, we choose $R=$ $100 \mathrm{Mbps}$ ). However, the latency component must be measured by injecting packets at low rates because $T$ models non-queueing delays incurred at the switch [3].

\subsection{Network-Calculus-Based Analysis}

In practical AFDX design, whether real-time requirements for all the time-critical tasks can be satisfied must be carefully examined. However, network-calculus-based analysis using measurement-based models only for source end systems and Ethernet switches may still yield overly pessimistic bounds (see Figure 1). To resolve this issue, we integrate measurement-based intermediate arrival curves (IACs) as follows: For algorithms relying on repeatedly applying networkcalculus theorems (e.g., [3]), measurement-based input and output arrival curves at Ethernet switches replace derived (or specificationbased) models. For optimization-based algorithms (e.g., [1]), extra constraints are added to further specify the traffic trajectory space using measurement-based curves.

\section{PRELIMINARY RESULTS}

We emulate an AFDX network shown in Figure 2a. We use netFPGA NICs to perform traffic capture and timestamp all the packets. Derived delay bounds are compared with observed maximum delays to help assess their quality (i.e., tightness). In our experiments, all source end systems generate packets with the same size, ranging from 64 bytes to 1500 bytes. Interfaces of all switches operate at 100 Mbps. We choose flow $f_{2}$ as our flow of interest. Note that in practical AFDX design, worst-case end-to-end delays for all time-critical flows should be evaluated.

As shown in Figure $2 \mathrm{~b}$, analytical bounds given by the approach in [1] with and without intermediate arrival curves (IACs) incorporated are indeed upper bounds of the end-to-end network-induced delays observed from measurements illustrated in box plot. Furthermore, integrating IACs does improve the quality (i.e., tightness)

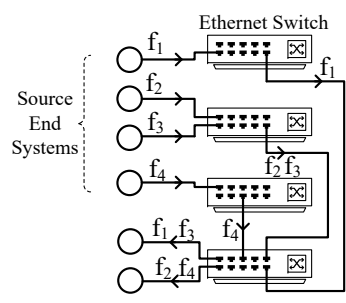

(a) AFDX network.

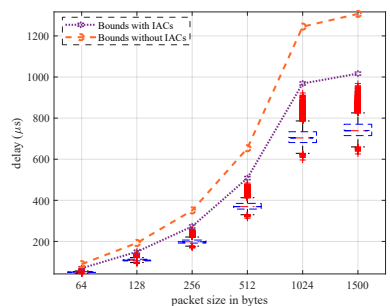

(b) Bounds vs. measurements.
Figure 2: Experiment settings and preliminary results. of application-specific delay bounds for AFDX systems, resulting in bounds that are only slightly greater than the worst-case delays observed. In fact, as shown in Figure 2a, flow $f_{2}$ travels through two switches to reach end system $e_{6}$. Intermediate arrival curves are measured before and after $f_{2}$ passes through the first and the second switches on its path. Through using the measured arrival curve at the input interface of an Ethernet switch, intrinsic pessimism of network-calculus traffic aggregation (i.e., the arrival curve of a flow aggregated from multiple sub-flows is simply the sum of those of the sub-flows) is effectively removed, and characteristics of aggregated traffic flows are accurately taken into account. At the output end of an Ethernet switch, serialization effect is modeled through the use of measured output arrival curve, which has a reduced burstiness component and will thus lead to a tighter input flow (or sub-flow) model for downstream Ethernet switch(es).

\section{CONCLUSION AND FUTURE WORK}

We propose to improve the quality of delay bounds obtained from network-calculus-based analysis through measurement-based modeling. Our preliminary results show that the combination of measurements and network calculus can generate sufficiently tight and formally provable bounds for AFDX networks, making it a valuable tool for deterministic delay performance analysis of AFDX systems. As our future work, we will explore establishing sophisticated network-calculus models (i.e., piece-wise linear arrival and service curves) using measurements and further study AFDX networks with other packet scheduling policies, such as round robin [2].

\section{ACKNOWLEDGMENTS}

This work is supported by NSF Award No. 1646458. Any opinions, findings, and conclusions or recommendations expressed in this paper are those of the author(s) and do not necessarily reflect the views of the sponsors of the research.

\section{REFERENCES}

[1] Anne Bouillard and Giovanni Stea. 2015. Exact Worst-Case Delay in FIFOMultiplexing Feed-Forward Networks. IEEE/ACM Trans. Netw. 23, 5 (October 2015), 1387-1400.

[2] A. Soni, X. Li, J. Scharbarg, and C. Fraboul. 2018. Optimizing Network Calculus for Switched Ethernet Network with Deficit Round Robin. In 2018 IEEE Real-Time Systems Symposium (RTSS). 300-311.

[3] Huan Yang, Liang Cheng, and Xiaoguang Ma. 2017. Analyzing Worst-Case Delay Performance of IEC 61850-9-2 Process Bus Networks Using Measurements and Network Calculus. In Proceedings of the Eighth International Conference on Future Energy Systems (e-Energy '17). 12-22.

[4] Noa Zilberman, Yury Audzevich, Georgina Kalogeridou, Neelakandan ManihattyBojan, Jingyun Zhang, and Andrew Moore. 2015. NetFPGA: Rapid Prototyping of Networking Devices in Open Source. In Proceedings of the 2015 ACM Conference on Special Interest Group on Data Communication (SIGCOMM '15). 363-364. 